\title{
Experimental study on abnormal thyroid function in patients with Hashimoto's thyroiditis caused by interference of thyroid hormone autoantibodies
}

\author{
Xiaofang Zhang ${ }^{1 *}$, Wenli Feng ${ }^{2 *}$, Zuoliang Dong ${ }^{1}$ \\ ${ }^{1}$ Department of Clinical Laboratory, Tianjin Medical University General Hospital, Tianjin, China \\ ${ }^{2}$ Department of Endocrinology and Metabolism, Tianjin Medical University General Hospital, Tianjin, China \\ *Contributed equally
}

\begin{abstract}
Introduction: Thyroid hormone autoantibody (THAAb) is one of the important factors affecting thyroid function measurement. By analyzing the examination of a patient suffered with Hashimoto's thyroiditis, we sought to find a correct assessment method.

Material and methods: Radioimmunoassay, chemiluminescence immunoassay on an ADVIA Centaur XP system and Architect i2000sr platform, and electrochemiluminescence immunoassay on a Roche Cobas 601 system were used for detecting thyroid function. Polyethylene glycol (PEG) precipitation were performed to eliminate the influence of THAAbs.

Results: The results showed that the patient's thyroid function was consistent with the clinical manifestations and conformed to the law of the hypothalamic-pituitary-thyroid axis at Architect-i2000sr platform and Roche-Cobas-601 system. The content of FT4 was significantly reduced and lower than the normal reference range, after the patients' serum was treated with PEG, which was in line with the clinical practice. The serum THAAb titer of the patients was nearly 100 times higher than that of the control group.

Conclusions: Considering an abnormal thyroid function examination, it is necessary for laboratory staff to retest samples on different platforms. It is of great significance to provide a true and accurate result to clinicians and patients. (Endokrynol Pol 2021; 72 (6): 668-669) Key words: Hashimoto's thyroiditis; thyroid function; thyroid hormone autoantibody; free thyroxine 4
\end{abstract}

\section{Introduction}

Autoantibodies are one of the disturbing factors in clinical practice [1] and often present in patients with autoimmune thyroid diseases. They directly target different antigens and exhibit nonspecific binding to markers in immunoassays. Thyroid hormone autoantibodies (THAAbs) affect the authenticity of thyroid hormone test results, leading to misdiagnosis or inappropriate treatment, and with unpredictable medical risks. The frequency of THAAbs is 1.8\% in the healthy adults [2], while the incidence is as high as $30-40 \%$ in patients with autoimmune thyroid disease [3, 4]. Increasing the awareness of the above problem is necessary to avoid reporting incorrect test results. Here, we studied a patient with a falsely elevated test result due to the presence of serum THAAbs. We attempted to identify a method to correctly evaluate the thyroid function status in THAAb-positive patients.

\section{Material and methods}

A 61-year-old woman presented with fatigue and oedema for 4 years. She took methimazole because of detecting elevated free thyroxine (FT4) and thyroid-stimulating hormone (TSH) levels in another hospital. The patient's main manifestations were fatigue, weakness, chills, oedema, and constipation. After repeated examination, except the abnormal $\mathrm{T} 4$ and pituitary thyrotropin, the results of our hospital were consistent with the previous tests.

Thyroid function tests (TFTs) [TSH, free triiodothyronine (FT3), and FT4] and the serum concentrations of total T3 and total T4 were measured by radioimmunoassay (RIA), chemiluminescence immunoassay on an ADVIA Centaur XP system and Architect i2000sr platform, and electrochemiluminescence immunoassay on a Roche Cobas 601 system, respectively.

For the autoantibody binding test, radiolabelled T3 or T4 was added to the serum of a patient and the mixed serum of a normal subject. Subsequently, equal volumes of $25 \%$ polyethylene glycol (PEG) solution were added to the samples after incubated for $2 \mathrm{~h}$ at $37^{\circ} \mathrm{C}$. Then, the mixture was centrifuged $\left(4^{\circ} \mathrm{C}, 10,000 \mathrm{rpm}\right)$ for $20 \mathrm{~min}$, and the radioactivity of the precipitate was determined. The presence of antibodies against T3 or T4 was determined in accordance with the ratio of the count of radioactivity to the total radioactivity $(\mathrm{B} / \mathrm{T})$. The concentrations of $\mathrm{T} 3, \mathrm{~T} 4$, and $\mathrm{TSH}$ in the PEG-treated supernatant were determined.

The patients' serum samples and 20 normal subjects were added with radiolabelled T3 and T4 analogues. The titres of anti-T3 and -T4 autoantibodies were determined through RIA. Nonspecific binding was subtracted from all sample readings, and the final percentage of $\left[{ }^{125} \mathrm{I}\right] \mathrm{T} 4$ or $\left[{ }^{125} \mathrm{I}\right] \mathrm{T} 3$ binding was calculated. The result was expressed as the antigen-antibody binding rate.

\section{Results}

TFT of a patient diagnosed with Hashimoto's thyroiditis on the ADVIA Centaur XP system revealed an increased 
Table 1. Comparison of the TFT results of different detection platforms

\begin{tabular}{lccccccccc}
\hline Test item & SI unit & Siemens & RR & RIA & RR & Abbott & RR & Roche & RR \\
\hline Total T3 & $\mathrm{nmol} / \mathrm{L}$ & 1.01 & $0.92-2.79$ & Not tested & default & 0.98 & $0.89-2.44$ & 1.35 & $1.30-3.10$ \\
\hline Total T4 & $\mathrm{nmol} / \mathrm{L}$ & 49.88 & $58.10-140.6$ & Not tested & default & 54.34 & $62.68-150.84$ & 61.21 & $66.00-181.00$ \\
\hline Free T3 & $\mathrm{pmol} / \mathrm{L}$ & 0.49 & $3.50-6.50$ & 0.63 & $2.07-6.0$ & 0.98 & $2.63-5.70$ & 0.68 & $3.10-6.80$ \\
\hline Free T4 & $\mathrm{pmol} / \mathrm{L}$ & 92.93 & $11.50-23.50$ & 2.63 & $6.65-19.9$ & 7.33 & $9.01-19.05$ & 9.56 & $12.00-22.00$ \\
\hline TSH & $\mathrm{mlU} / \mathrm{L}$ & $>150$ & $0.30-5.00$ & $>81$ & $0.7-7.59$ & $>100$ & $0.35-4.94$ & $>100$ & $0.27-4.20$ \\
\hline
\end{tabular}

$\mathrm{RR}$ — reference range; T3 — triiodothyronine; T4 — thyroxine; TSH — thyroid-stimulating hormone

serum FT4 and TSH, and a reduced FT3. These results did not conform to the law of hypothalamic-pituitary-thyroid axis and the clinical manifestations. Surprisingly, the results of TT3 and TT4 were consistent with the clinical features. Subsequently, besides detecting FT3/FT4/TSH through RIA, both FT3/FT4/TSH and T3/T4 levels were detected with the Architect i2000sr platform and Roche Cobas 601 system. The results were in accordance with regular rules. As shown in Table 1, the FT3 and TSH results from the four different detection systems show consistent trends, and the FT4 results are clinically consistent with the results of most platforms, except for the Siemens detection system.

Specific binding to $\mathrm{I}^{125}$-T4 was observed in the patient's serum. However, nonspecific binding to $\mathrm{I}^{125}-\mathrm{T} 4$ was also found in the serum of normal subjects. The former was 100-times higher than the latter. PEG precipitation, a most commonly used immunosubtraction method for the detection of macroprolactin and analytes, was used to precipitated the serum of the patient. TFT showed FT3 and TSH levels did not change significantly, whereas FT4 levels decreased significantly. These trends were consistent with the clinical manifestations of the patient (Supplementary File - Tab. 1).

The THAAb titre of the patients was more than 100-times that of the control group. The mean value of the T3 antibody (\%) in the normal control group was $2.1 \%$ and that in the patient was $4.7 \%$, which was within the normal range. The mean value of the $\mathrm{T} 4$ antibody $(\%)$ in the normal control group was $0.5 \%$ and that in the patients was $59 \%$, which was considerably higher than the normal level.

\section{Discussion}

Potential causes of spurious TFT results include nonspecific binding of endogenous circulating factors such as heterophilic antibodies, albumin variants, and THAAbs [7]. THAAb, an analyte autoantibody specific to T4 and T3, is the only antibody reported to interfere with TFT.

Some studies recommend PEG precipitation to eliminate the interference of THAAbs in serum TFT.

When TFT results are inconsistent in clinical testing, other methods for investigating immunoassay interference include the following: (a) demonstrating nonlinear response to sample dilution; (b) demonstrating iodothyronine binding to the patient's IgG through electrophoresis or precipitation with anti-IgG; (c) blocking heterophile antibodies with nonimmune serum or blocking tubes; and (d) suppressing patient antibodies with immunosuppressive therapy [9].

In this case, we confirmed immunoassay interference by repeating the analysis on different platforms and demonstrating the presence of THAAbs through PEG precipitation.

\section{Funding}

The work was supported by National Natural Science Foundation of China grants (81402391).

\section{Conflicts of interest}

The authors declare that they have no conflict of interest.

\section{Authors' contribution}

All authors designed, analysed, wrote the draft of the manuscript, and approved the final version for publication. All authors agree to accountability for the accuracy and integrity of the work.

\section{Ethical approval}

This study was approved by the Ethics Committee of Tianjin Medical University General Hospital. The subject had given written informed consent.

\section{References}

1. Kricka L. Interferences in Immunoassay-Still a Threat. Clin Chem. 2000; 46(8): 1037-1038, doi: 10.1093/clinchem/46.8.1037, indexed in Pubmed: 10926879.

2. Sakata S, Matsuda M, Ogawa T, et al. Prevalence of thyroid hormone autoantibodies in healthy subjects. Clin Endocrinol (Oxf). 1994; 41(3): 365-370, doi: 10.1111/j.1365-2265.1994.tb02558.x, indexed in Pubmed: 7955443.

3. Sakata S, Nakamura S, Miura K. Autoantibodies against thyroid hormones or iodothyronine. Implications in diagnosis, thyroid function, treatment, and pathogenesis. Ann Intern Med. 1985; 103(4): 579-589, do i: 10.7326/0003-4819-103-4-579, indexed in Pubmed: 3898955.

4. Després N, Grant A. Antibody interference in thyroid assays: a potential for clinical misinformation. Clin Chem. 1998; 44(3): 440-454, doi: 10.1093/clinchem/44.3.440, indexed in Pubmed: 9510847.

5. Lee MiNa, Lee SY, Hur KY, et al. Thyroxine (T4) Autoantibody Interference of Free T4 Concentration Measurement in a Patient With Hashimoto's Thyroiditis. Ann Lab Med. 2017; 37(2): 169-171, doi: 10.3343/alm.2017.37.2.169, indexed in Pubmed: 28029007.

6. Jones AM, Honour JW. Unusual results from immunoassays and the role of the clinical endocrinologist. Clin Endocrinol (Oxf). 2006; 64(3): 234-244, doi: 10.1111/j.1365-2265.2006.02439.x, indexed in Pubmed: 16487430. 